ZEINS

\title{
The Fast mode for ZEISS LSM 880 with Airyscan: high- speed confocal imaging with super-resolution and improved signal-to-noise ratio
}

In May 2016, ZEISS announced the next innovation step for laser-scanning microscopy imaging with the introduction of the Fast mode for ZEISS LSM 880 with Airyscan. The new concept combines the Airyscan pinhole-plane detection technology with a new illumination shaping approach, enabling a fourfold increase in image acquisition rates. With the new Fast mode, Airyscan now affords researchers simultaneous access to super-resolution, increased signal-to-noise ratio and increased acquisition speeds without compromise.

The confocal laser-scanning microscope (LSM) has become one of the most popular instruments in basic biomedical research for fluorescence imaging. The main reason laser-scanning microscopy has become so popular is that the technique provides researchers with high-contrast images and a versatile optical sectioning capability to investigate three-dimensional biological structures ${ }^{1}$. The optical sectioning ability of an LSM is a product of scanning a diffraction-limited spot across a sample to create an image one point at a time. Traditionally, the generated fluorescence for each point is collected and focused back through an aperture (pinhole) onto a unitary detector (typically a photomultiplier tube $(\mathrm{PMT}))$. The traditional detection scheme allows the pinhole to reject the out-of-focus light and the PMT to turn the remaining light from the focal plane into a digital signal to form an optical sectioned image ${ }^{2}$. As confocal systems have developed over the past 25 years, different innovations have provided the LSM with the flexibility to address research applications in many areas, providing data on cell differentiation, cell tracking, live cell kinetics, protein expression, protein localization, neural network connectivity, tissue structure, gene expression and protein/ gene function (this list is not meant to be inclusive). For most application areas the LSM is well suited to provide the needed optical sectioning capability for observing fast processes in a 3D structure. However, until the introduction of the Fast mode for Airyscan $^{3}$, researchers were forced to compromise on resolution or signal-to-noise ratio (SNR) to achieve the required temporal resolution.

\section{Joseph Huff}

Carl Zeiss Microscopy, LLC, Thornwood, New York, USA. Correspondence should be addressed to J.H. (joseph.huff@zeiss.com).
Improving acquisition speeds on a point-scanning confocal microscope

The acquisition speed of a conventional LSM is determined by how fast a single diffraction-limited laser spot can be moved across a desired field of view with a desired pixel count. Therefore, to increase the achievable scan speeds of a conventional LSM, a researcher must decrease the amount of time the excitation laser spends on each pixel (pixel dwell time), reduce the number of pixels or reduce the image field of view. As a result, when using a conventional LSM a researcher must compromise on image SNR by reducing pixel dwell time, on spatial resolution by reducing the pixel count or on field of view by zooming in to a portion of the structure of interest. Traditionally, for point-scanning LSMs reduction of the pixel dwell time has been the preferred approach to maximize scan speeds in the form of resonant scanning.

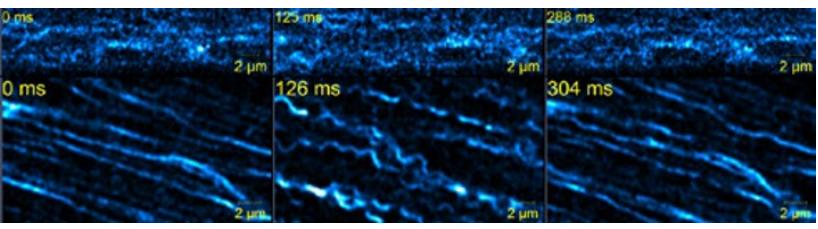

Figure 1 | Application example of a resonant scanning time series compared with a time series obtained with the Airyscan Fast acquisition mode using cardiomyocyte cells with tubulin-EMTB to measure microtubule buckling at high frame rates. In each image, the top portion shows a still image from a resonant scanning time series acquired at 80 frames per second, and the bottom, portion shows a still image from an Airyscan Fast mode time series acquired at 96 frames per second. The images from left to right show the resting tubules, contracted tubules and tubules returned to the resting state after contraction. Images courtesy of B. Prosser (University of Pennsylvania). 
Resonant scanning uses a sinusoidal scanning approach that varies the pixel dwell time across the field of view in order to achieve a maximum frame rate of around $30 \mathrm{~Hz}$ for a full field of view. When the laser spot is scanned faster across the field of view, the pixel dwell time is shortened and, consequently, the amount of time per pixel spent collecting fluorescence is also decreased, which affects the resulting SNR of the image. As the acquisition speed increases, fewer and fewer photons are available, resulting in deterioration of the image SNR. The outcome is not only a noisy image but also compromised spatial resolution in which fine structures cannot be properly resolved (Fig. 1, upper panel). To compensate for the deteriorating SNR, the laser power can be increased, but this too has disadvantages: the danger of bleaching the fluorophore and/ or damage to live samples from phototoxic effects (for example, free oxygen radicals) becomes more prevalent at higher laser powers, and thus the risk of influencing experimental outcomes is increased $^{4-6}$. Therefore, traditional techniques to improve image acquisition speeds demand that a researcher compromise image SNR, resolution, field of view and laser exposure, and the effects on all of these are likely to impede the research goal.

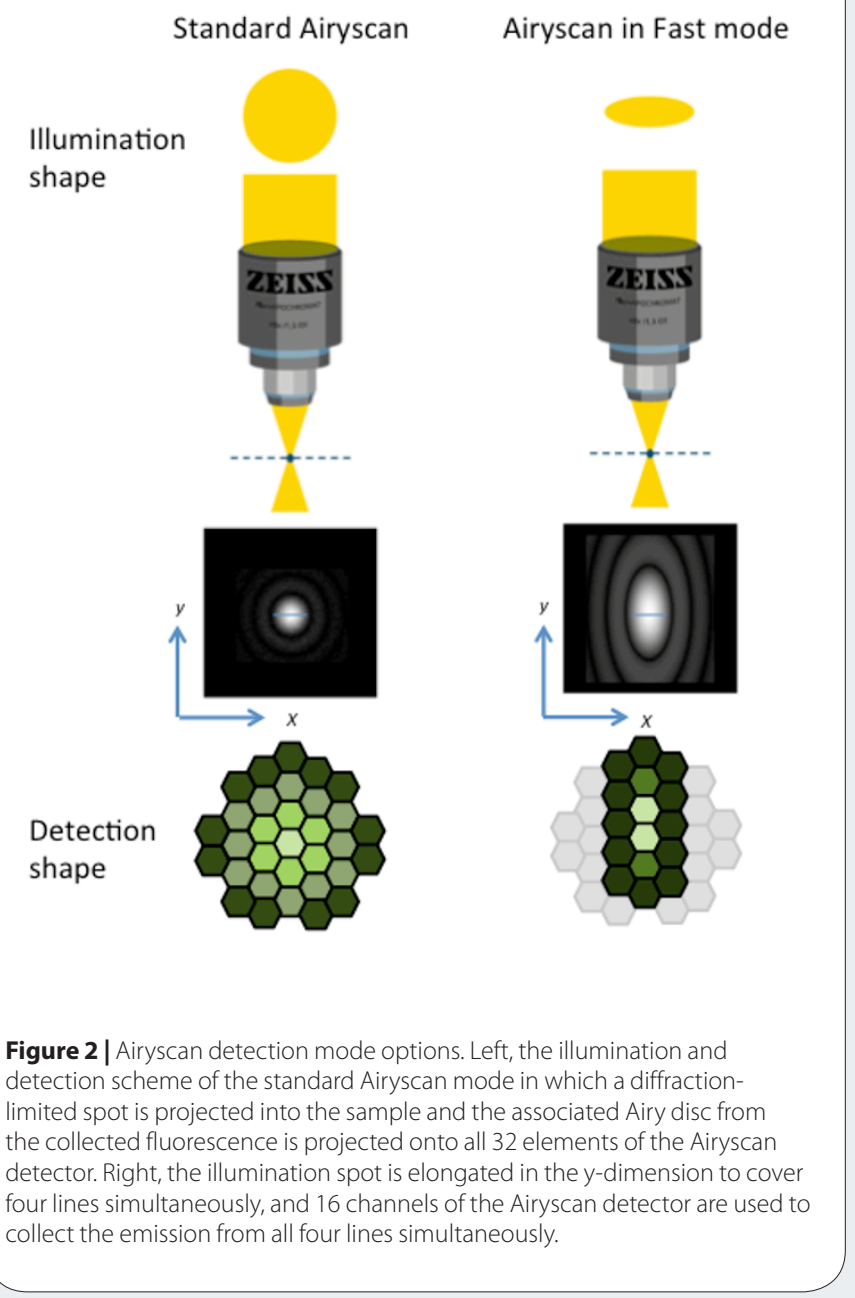

\section{The Fast mode for Airyscan}

To overcome the trade-off between acquisition speed and image SNR, ZEISS has combined the Airyscan pinhole-plane detection concept with an illumination shaping approach for a new fast acquisition mode. With the Fast mode for Airyscan, the excitation spot is elongated along the $y$-axis to cover four lines in a single scan (Fig. 2). Through the use of a parallelization approach, acquisition speeds are increased by a factor of four while high pixel dwell times are maintained, resulting in a high image SNR. Hence, although the imaging speed is notably increased, high SNRs can be preserved, resulting in meaningful data. Moreover, because the Fast mode is based on the Airyscan detector, the additional, simultaneous increases in SNR and resolution are retained. Therefore, Airyscan in Fast mode offers a researcher the unprecedented combination of a $1.5 \times$ increase in resolution and a $4 \times$ increase in SNR at four times the acquisition speed (Fig. 1, lower panel).

\section{Summary}

In summary, the Airyscan detection module dispenses with the classical physical pinhole and unitary detector assembly and uses a new pinhole-plane image detection assembly based on a novel 32-channel GaAsP-PMT area detector. In 2014, Airyscan was implemented to provide a flexible and robust method to substantially increase resolution and SNR compared with those achieved with a traditional confocal LSM ${ }^{3,7,8}$. Using the Airyscan detector design in the innovative Fast mode provides a substantial increase in speed while simultaneously providing increases in resolution and SNR relative to traditional confocal LSMs.

1. Conchello, J.-A. \& Lichtman, J.W. Nat. Methods 2, 920-931 (2005).

2. Neu, T.R. \& Lawrence, J.R. Trends Microbiol. 23, 233-242 (2015).

3. Huff, J. Nat. Methods 12, i-ii (2015).

4. Wäldchen, S., Lehmann, J., Klein, T., van de Linde, S. \& Sauer, M. Sci. Rep. 5, 15348 (2015).

5. Li, D. et al. Science $\mathbf{3 4 9}$, aab3500 (2015).

6. Kucsko, G. et al. Nature $\mathbf{5 0 0}, 54-58$ (2013).

7. Weisshart, K. ZEISS Technology Note: The Basic Principle of Airyscanning (Carl Zeiss Microscopy, 2014)

8. Huff, J., Bathe, W., Netz, R., Anhut, T. \& Weisshart, K. ZEISS Technology Note: The Airyscan Detector from ZEISS. Confocal Imaging with Improved Signal-to-noise Ratio and Superresolution (Carl Zeiss Microscopy, 2015).
This article was submitted to Nature Methods by a commercial organization and has not been peer reviewed. Nature Methods takes no responsibility for the accuracy or otherwise of the information provided. 